\title{
S-MIM field trials results
}

\author{
Romain Hermenier ${ }^{1, *, \dagger}$, Alessandro Del Bianco ${ }^{1}$, Maria-Antonietta Marchitti ${ }^{1}$, \\ Thomas Heyn ${ }^{2}$, Annamaria Recchia ${ }^{3}$, Florian Collard ${ }^{3}$, Marco Andrenacci ${ }^{4}$, \\ Gaeteno Mendola ${ }^{4}$ and Attilio Vaccaro ${ }^{4}$ \\ ${ }^{I}$ DLR, Oberpfaffenhofen, Wessling, Germany \\ ${ }^{2}$ Fraunhofer Institute for Integrated Circuits - IIS, Erlangen, Germany \\ ${ }^{3}$ Eutelsat, Paris, France \\ ${ }^{4} \mathrm{MBI}$, Pisa, Italy
}

\begin{abstract}
SUMMARY
An intelligent transport system open platform integrating the S-band Mobile Interactive Multimedia messaging return channel protocol over satellite (based on Enhanced Spread Spectrum Aloha) has been developed and tested under real environment conditions within the framework of SafeTRIP, an FP7 EU-funded project. This paper presents the first field trials results using the S-band Mobile Interactive Multimedia technology. The introduced forward and return link outcomes have been derived from mobile field trials carried out in the surroundings of the German Aerospace Center (DLR) in Germany. Finally the validation of the system performances has been realized thanks to the use of a traffic emulator that can simulate a large population of Enhanced Spread Spectrum Aloha terminals. Copyright (C) 2014 John Wiley \& Sons, Ltd.
\end{abstract}

Received 6 November 2012; Revised 4 November 2013; Accepted 22 November 2013

KEY WORDS: S-MIM; field trials; DVB-SH; E-SSA; S-band; link layer; power control

\section{INTRODUCTION}

The newly ETSI standardized S-band Mobile Interactive Multimedia (S-MIM) protocol [1] describes an innovative satellite/terrestrial communication system capable of efficiently use the available S-band allocation for pan-European Mobile Satellite Services. Providing various services such as broadcast, messaging and bidirectional communications, this protocol relies on the Digital Video Broadcasting - Satellites services to Handhelds (DVB-SH, [2]) in the forward link and on two different interfaces in the return link: quasi-synchronous code division multiple access [3] that requires a synchronous access and Enhanced Spread Spectrum Aloha (E-SSA, [4-6]) that requires an asynchronous access. More exhaustive insights of the S-MIM protocol can be found in the standard [1] as well as in [7] and [8], which present overviews of the S-MIM specifications.

Within the framework of the SafeTRIP project [9], an intelligent transport system open platform integrating the S-MIM protocol has been developed and tested under real environment conditions. This in field evaluation of the platform represents the first on air test of the S-MIM technology, although the E-SSA wave form has already been validated on the field [10]. With respect to these preliminary field trials of the E-SSA wave form, the S-MIM link layer (based on the Return Link Encapsulation (RLE) protocol [11]) has been developed and integrated in the open platform, providing thus statistics at link layer and Internet Protocol (IP) levels.

Focusing on the messaging part of the technology for the return link (E-SSA), this paper gives an overview of the first S-MIM field trials outcomes with a strong emphasis on the achieved link layer performances. The latter have also been validated under heavy traffic load conditions thanks to the

*Correspondence to: R. Hermenier, DLR, Oberpfaffenhofen, Wessling, Germany.

${ }^{\dagger}$ E-mail: romain.hermenier@dlr.de 
use of a traffic emulator simulating a large population of E-SSA terminals. Furthermore, an analysis of the DVB-SH reception during the field trials is also provided so as to check the reliability of the forward link received signal. Finally, a potential use of the S-MIM technology for end-users applications is shortly discussed: the SafeTRIP platform is presented and examples of applications exploiting the S-MIM technology and developed during the project are illustrated.

The rest of the paper is organized as follows. Section 2 introduces the system setup and details the most relevant components of the open platform. Section 3 describes the field trials results, which represent our main contribution. The SafeTRIP platform is presented in Section 4, while Section 5 draws the conclusions.

\section{SYSTEM SETUP}

The system setup used during the trials is composed of different segments. First of all it comprises the user segment, which corresponds to the mobile terminal prototype. The latter, integrated in a vehicle, comprises the S-MIM link layer, the E-SSA modulator, the DVB-SH demodulator and the reception $(\mathrm{Rx})$ and transmission (Tx) antennas. The overall mobile prototype is dubbed as the On-Board Unit (OBU). Then the geostationary (GEO) satellite Eutelsat 10A [12], equipped with the S-band payload represents the space segment. Finally, the satellite ground hub located at Rambouillet teleport (France) constitutes the ground segment. Various modules are implemented in the hub such as the E-SSA demodulator, the S-MIM link layer with all the control plane functionalities and the DVB-SH modulator. Furthermore a traffic emulator, able to emulate a large population of E-SSA terminals, is also integrated in the hub for validation purposes. Figure 1 illustrates a simplified version of the overall system architecture used during the field trials.

This satellite-based system can offer full European coverage without any Complementary Ground Components (CGC). To further increase the capacity in dense populated areas CGCs can be added. No CGC have been used for the field trials to demonstrate the performance for a satellite only system.

\subsection{The user segment}

The mobile prototype developed in [9] and evaluated during the field trials is based on an industrial PC platform (DT4900) and is composed of three different modules, namely the DVB-SH receiver, the S-MIM link layer software and the E-SSA modulator prototype (Figure 2). The latter, developed by Fraunhofer and compliant to [1, part 3] comprises the following parts:

- Part of the modulator functional blocks (turbo encoder) is implemented as software modules, running on the PC.

- The modulator firmware running on a Field Programmable Gate Arrays transforms the received turbo coded words from the PC into the digital transmitter output signal by performing framing, spreading, preamble insertion and pulse shaping.

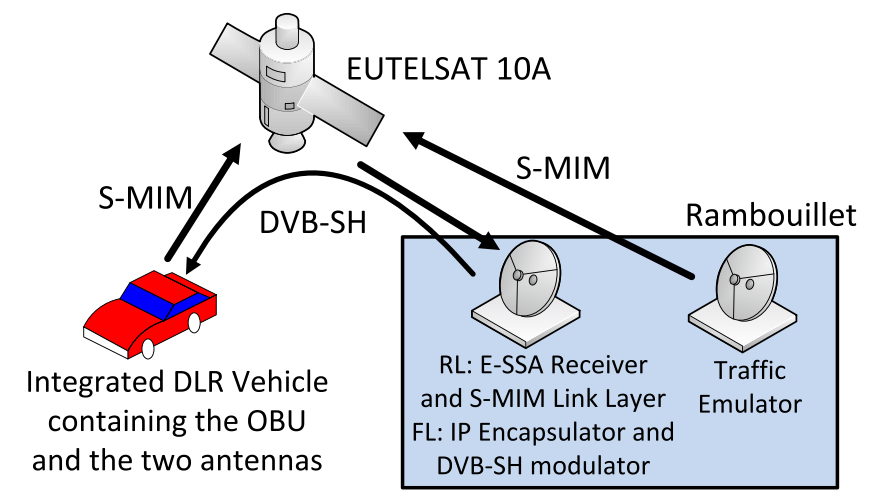

Figure 1. System architecture for the field trials. S-MIM, S-band Mobile Interactive Multimedia; DVB-SH, Digital Video Broadcasting - Satellite services to Handhelds; E-SSA, Enhanced Spread Spectrum Aloha; RL, Regular Latency; FL, forward link; IP, Internet Protocol; OBU, On-Board Unit. 


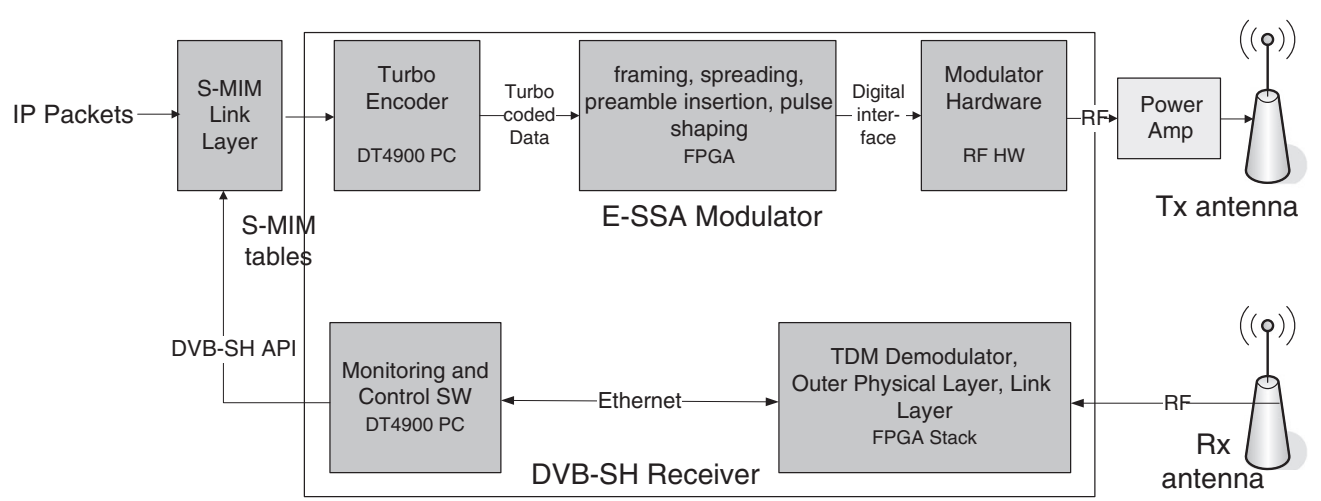

Figure 2. Block diagram of the mobile prototype. S-MIM, S-band Mobile Interactive Multimedia; IP, Internet Protocol; E-SSA, Enhanced Spread Spectrum Aloha; FPGA, Field Programmable Gate Arrays; RF, radio frequency; HW, hardware; Tx, transmission; Rx, reception; DVB-SH, Digital Video Broadcasting - Satellite services to Handhelds; SW, software; TDM, time-division multiplexing

- The modulator hardware transforms the digital output signal into the analogue radio frequency (RF) signal in S-band to be provided to the power amplifier.

The S-MIM link layer software for messaging return channel has been developed and tested by DLR. Although the software does not include all the S-MIM features, such as header compression or encryption, the main characteristics have been implemented. Figure 3 shows a simplified block diagram of the OBU link layer. IP packets coming from higher layers are first scheduled depending on the targeted class of service (1 or 2), which specifies two different levels of priority. Scheduled packets are then fragmented using the RLE algorithm and transmitted to the E-SSA modulator through the automatic repeat request mechanism. The latter manages different parameters such as the timing to transmit the fragment, the number of retransmission, the acknowledgment time-out and bases its behavior on the received parameters from the signaling tables. The transmission power control and the related control plane functionalities, such as load control, are received from the forward link through the S-MIM tables.

The satellite receiver as used for the trials is compliant to the DVB-SH standard [2]. The receiver is based on a demodulator IP core development ready for the integration in an application-specific integrated circuit. The IP core supports all profiles of DVB-SH. The profile Satellite services to

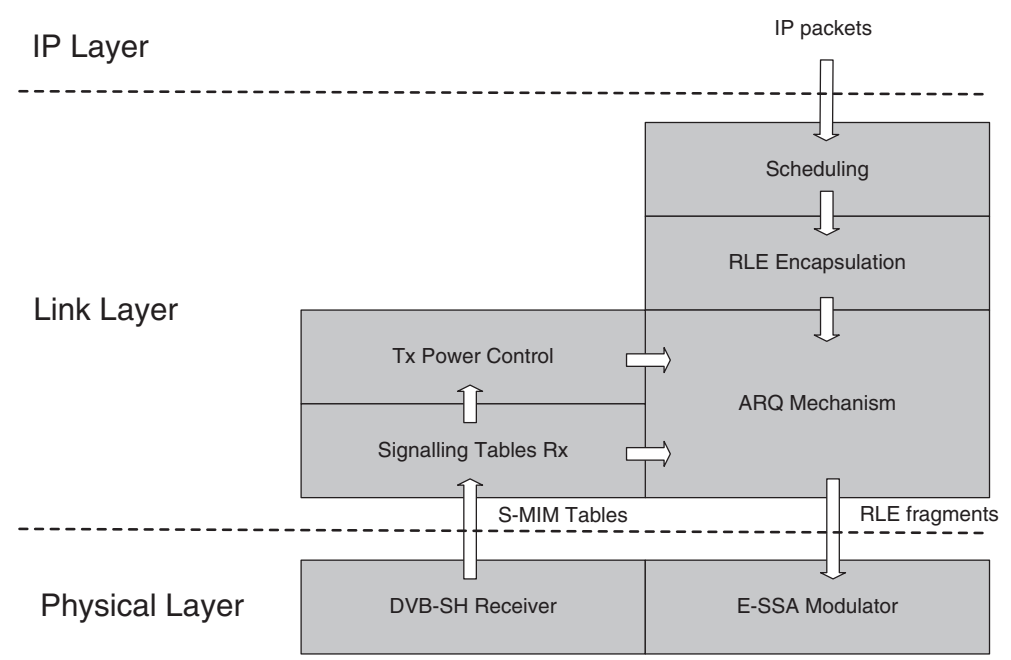

Figure 3. Block diagram of the On-Board Unit link layer. IP, Internet Protocol; RLE, Return Link Encapsulation; ARQ, automatic repeat request; Tx, transmission; Rx, reception; S-MIM, S-band Mobile Interactive Multimedia; E-SSA, Enhanced Spread Spectrum Aloha. 
Handhelds - profile B (SH-B) (time-division multiplexing (TDM) for satellite, OFDM for terrestrial $\mathrm{CGC}$ ), class 2 (long physical layer time interleaving) was used for the field trials (the supported DVB-SH operation modes SH-A, SH-B, as well as classes 1 and 2 are explained in [2]). This IP core has been extended in the SafeTRIP project to support the optional Low Latency Extension as described in the DVB-SH standard [2]. The demodulator IP was mapped to a modular Field Programmable Gate Arrays based field-test receiver platform, called embedded module, which is integrated in the DT4900 PC platform together with the E-SSA equipment. The functional blocks and the hardware partitioning of the DVB-SH receiver are depicted in Figure 4. The embedded module consists of a stack of four PCBs used to implement the SafeTRIP DVB-SH receiver. Baseline is to receive one $2.5 \mathrm{MHz}$ band transmitted via satellite and carrying a TDM signal. This signal may contain a low latency (LL) component having the same code rate as the regular latency (RL) component. Regarding the signal processing, the TDM demodulator output is forwarded to two boards for processing of the outer physical layer of the RL and LL streams, respectively. The uppermost PCB is configured to implement the link layer functions. In addition, the PCB incorporates a microcontroller environment executing the DVB-SH API software for configuring, controlling and monitoring the receiver as well as for delivery of the received data. This software has its counterpart running on the DT4900-CPU. The DVB middleware receives a data stream from the DVB-SH forward link. The contained data packets are used to extract information about available channels and other data from the PSI/SI tables or Electronic Service Guide packets. In the prototype implementation, static packet identifiers are used to carry the S-MIM signaling tables. To set up this data stream, the DVB middleware has to configure the DVB-SH receiver by using the DVB-SH API.

For the field trials, the OBU as well as the RF components have been integrated in a research test vehicle owned by DLR as illustrated in Figure 5. It must be noted that two different antennas for transmission and reception were used for the trials, although a prototype of one unique antenna is currently being completed.

\subsection{The space segment}

The satellite Eutelsat 10A is a GEO satellite, located at $10^{\circ}$ East. Apart from the Ku-band and C-band payload, it also features a major innovation by carrying an S-band payload enabling, for the first time in Europe, the delivery of mobile interactive multimedia services directly onto user mobile terminals and vehicles. The S-band payload is the one used to carry out the first S-MIM trials [10]. It operates in forward mode in the range $2185-2200 \mathrm{MHz}$ and in return mode, in the range 1995-2010 MHz. In its

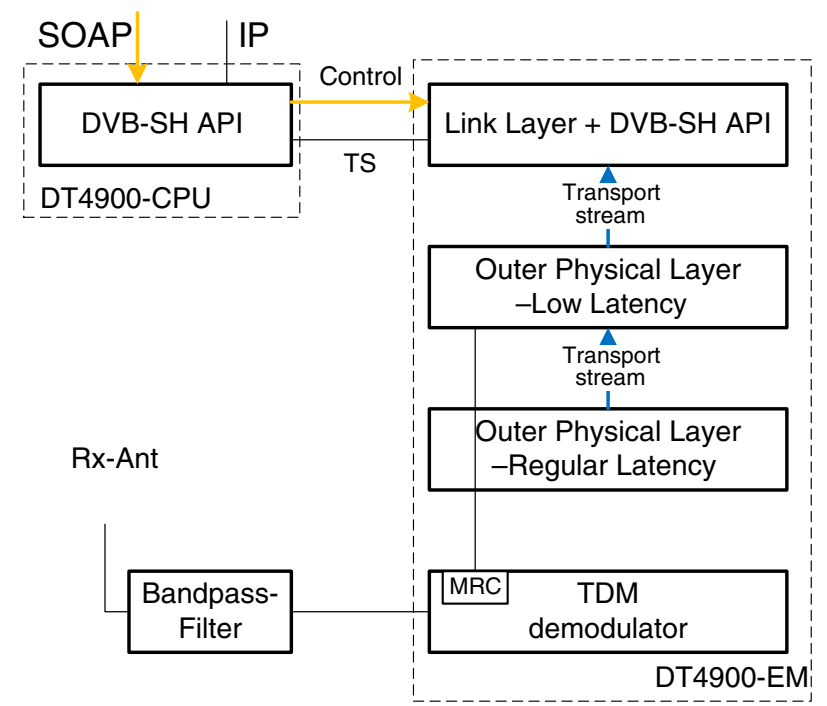

Figure 4. Functional blocks and hardware partitioning of the DVB-SH receiver. SOAP, Simple Object Access Protocol; IP, Internet Protocol; DVB-SH, Digital Video Broadcasting - Satellite services to Handhelds; Rx, reception; TDM, time-division multiplexing. 


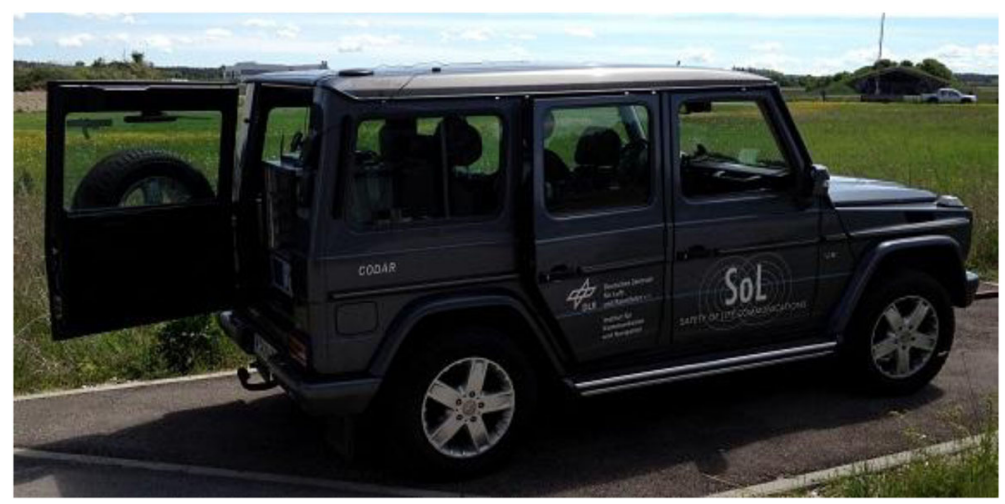

Figure 5. DLR integrated test vehicle (the On-Board Unit is installed in the trunk, and the two antennas are located on the roof of the vehicle).

current configuration, it delivers coverage of the Europe from two operational beams. Each beam is able to provide $5 \mathrm{MHz}$ of bandwidth capacity and operates in opposite circular polarization. While user-links are operated in S-band, the corresponding uplink and downlink are received by the hub in Ku-band. For more exhaustive insights, a complete link budget of the space segment is given in [13].

\subsection{The ground segment}

The ground segment, which constitutes a crucial point for the validation of the system performances, is made up of two major components, namely the satellite ground hub and the traffic emulator.

The ground hub comprises on one side the IP encapsulator with the DVB-SH modulator for the forward link services and on the other side the E-SSA receiver with the link layer software. The latter was developed by DLR and aims at reordering, for each user, the fragments coming from the E-SSA demodulator, performs the RLE decapsulation and forwards the reassembled packet to the upper layer. At the same time, it has to monitor the channel load and send feedbacks to the terminals updating the S-MIM signaling tables forwarded through the forward link. MBI has completed the implementation of the first prototype of an E-SSA demodulator. The outcome of this activity is a software platform, called E-SSA test-bed made up of several modules. It can be used to demonstrate the S-band return channel both in a real scenario and in a laboratory. This test-bed is composed of the E-SSA traffic emulator, the E-SSA manager and demodulator and of radio frequency Tx/Rx front-ends. More exhaustive insights on the E-SSA test-bed and more particularly on the implementation of the E-SSA demodulator can be found in [14]. Finally, it is worth mentioning that the performances of the E-SSA demodulator are enhanced with the use of power randomization at the terminal side [1].

The traffic emulator can emulate an ETSI S-MIM compliant large population of E-SSA terminals (background traffic) with no traffic limitation by generating the samples of the signal which will be digital-to-analog converted by the Tx Software Defined Radio. Packets can be generated with a Poisson or constant distributions (interdelivery time). It gives an output signal at an intermediate frequency $(70 \mathrm{MHz})$. Then it is up-converted to reach the S-band frequencies assigned to Solaris Mobile. A high power amplifier is inserted before the parabolic antenna in order to emulate aggregated traffic up to 7000 transmitting terminals per second, each one with a fixed Effective Isotropically Radiated Power and signal-to-noise ratio (SNR) over the duration of each packet. In addition to the traffic emulator, a pilot generator transmits one reference packet per second over the uplink chain. The modulator is connected to a very accurate clock. The reference packet is needed at the hub side so as to compensate the frequency errors introduced by the whole chain (satellite Doppler). It is also necessary in order to center the demodulator frequency with an error less than $1 \mathrm{kHz}$, the upper bound of the tolerated frequency error beyond which performance degradation appears. The traffic emulator associated to the E-SSA demodulator is used to demonstrate the S-band return channel in a real scenario. 


\section{FIELD TRIALS RESULTS}

This section focuses on the validation of the forward and return link by carrying out field trials. For both cases, a unique route in southern Germany has been selected, as shown in Figure 6. The route has a length of $272 \mathrm{~km}$ and covers various Land Mobile Satellite (LMS) environments. Figure 7 illustrates the met LMS environments over the selected itinerary, namely Intermediate Tree Shadowing, Heavy Tree Shadowing, open area and suburban area. An estimation of the percentage of each LMS environment encountered is also reported in the legend of Figure 7.

\subsection{Forward link analysis}

The DVB-SH forward link has been validated by performing field trials (itinerary of Figure 6). Because the OBU prototype does not currently support simultaneous reception and transmission, the DVB-SH reception quality is affected by the outages caused during the E-SSA transmission bursts (further optimization of the RF components would however mitigate this self-interference). To quantify the DVB-SH QoS under realistic conditions, the trials have been performed with enabled E-SSA transmission

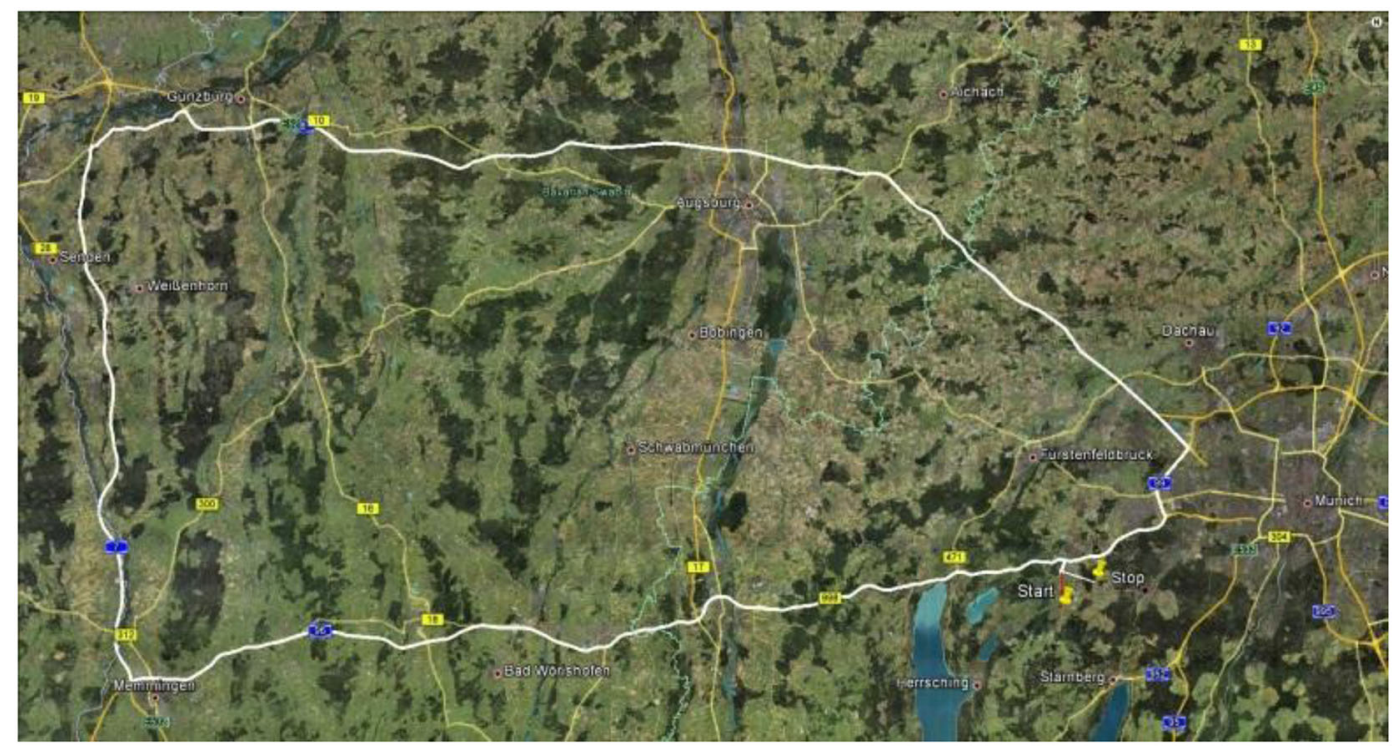

Figure 6. Field trials itinerary.

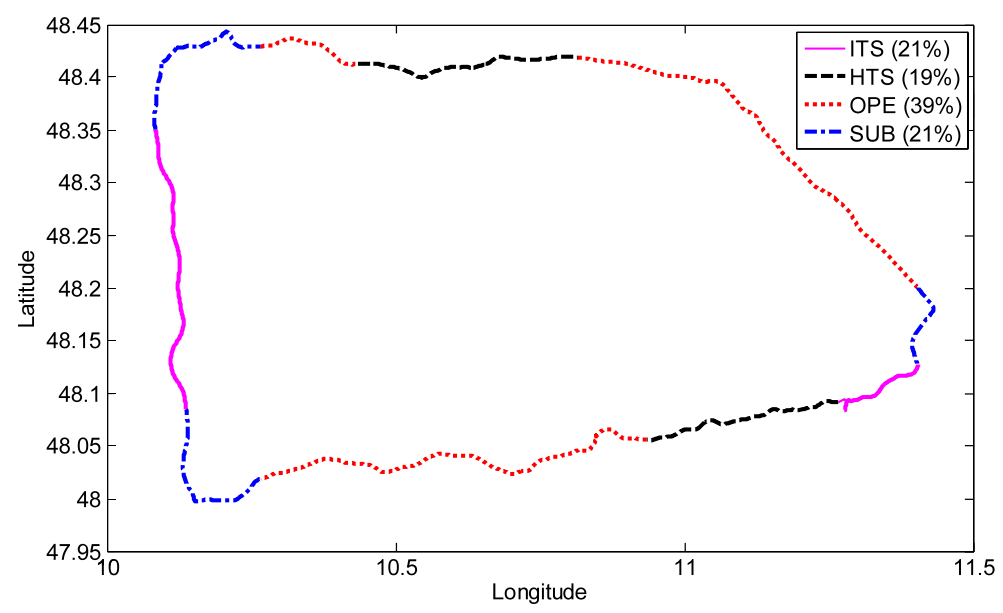

Figure 7. Land Mobile Satellite environments for the considered itinerary. ITS, Intermediate Tree Shadowing; HTS, Heavy Tree Shadowing; OPE, open area; SUB, suburban area. 
subsystem. The transmission parameters of the forward link signal have been as follows: The RF bandwidth has been $2.5 \mathrm{MHz}$, with Quadrature Phase-Shift Keying modulation and a code rate 1/3. A uniform time interleaving profile with length 10 s has been selected for the RL stream, whereas the LL stream has not been enabled in the hub uplink equipment.

This decision impacts both the forward and return links in different manners. As to the forward link, the use of the LL profile would allow a faster reception of the information (acknowledgments and signaling tables) but would degrade the Packet Error Rate as the transmitted packets over the forward link are not protected by the interleaver anymore. Instead, for the return link, the non-availability of the LL profile implies that the received signaling information from the forward link arrives with a 10s delay at the terminal side. The reception of delayed signaling information will cause a delayed reaction of the behavior of the terminal (e.g., if the hub requires the terminals to lower their data rates, this will be applied with a delay of 10s). However, these 10s delay do not impact the transmission decision at the physical layer as it only depends on the immediate received forward link carrier-to-noise ratio.

The key performance indicator considered for the forward link analysis is the ESR5(20) criterion. The latter as used in the DVB community is defined as the ratio of windows for which ESR5 is fulfilled, over the total number of windows. The ESR5 criterion is fulfilled when in a time interval of $20 \mathrm{~s}$ there is at most $1 \mathrm{~s}$ in error. The resulting time series of the received carrier-to-noise-and-interference ratios values is shown in Figure 8, visualizing the variations of the received signal depending on the current vehicle position. The resulting ESR5(20) vector over time as well as the time series of the erroneous seconds are displayed in Figure 9.

As to the measurement results, the following conclusions can be drawn. Even with the degradation of the simultaneous E-SSA transmissions, the forward link overall ESR5(20) ratio of 91\% is above the target QoS as specified in the DVB-SH Implementation Guidelines [15], where a minimum ESR5(20) of $90 \%$ is recommended. The corresponding word error rate has been only $4.4 \%$, so error free reception is achieved for a time share of $95.6 \%$. In our case, the 20s sliding window as applied to calculate the ESR5(20) criterion leads to a higher share of degraded intervals than indicated by the word error rate. As example, a short dropout of $2 \mathrm{~s}$ leads to 18 affected 20s intervals/windows, where at least two erroneous seconds occur.

Furthermore there are mainly two longer signal losses observed around 2300 and $8700 \mathrm{~s}$, causing the degradation of the total ESR5(20) ratio. Looking more closely into the data, the first signal loss is caused by a long stop behind a highway gas station blocking the satellite signal. The second loss was caused by stop-and-go traffic conditions on a highway in west-east direction in dense forest environment (Heavy Tree Shadowing causing satellite signal attenuation), together with E-SSA transmissions (causing an additional time share without satellite signal). For the rest of the route, the DVB-SH reception is excellent with only short outages after the forward error correction.

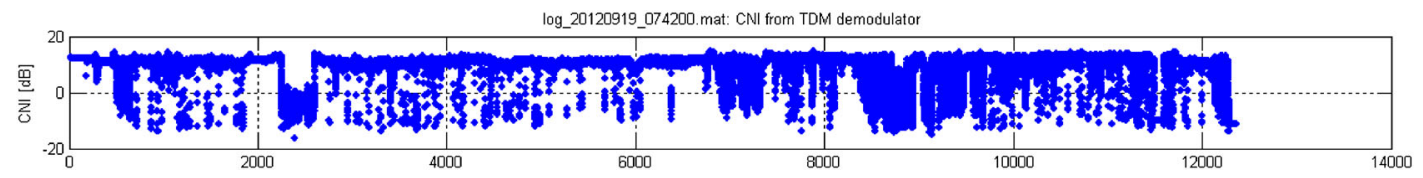

Figure 8. Carrier-to-noise-interference ratio (CNI) time series from the drive.
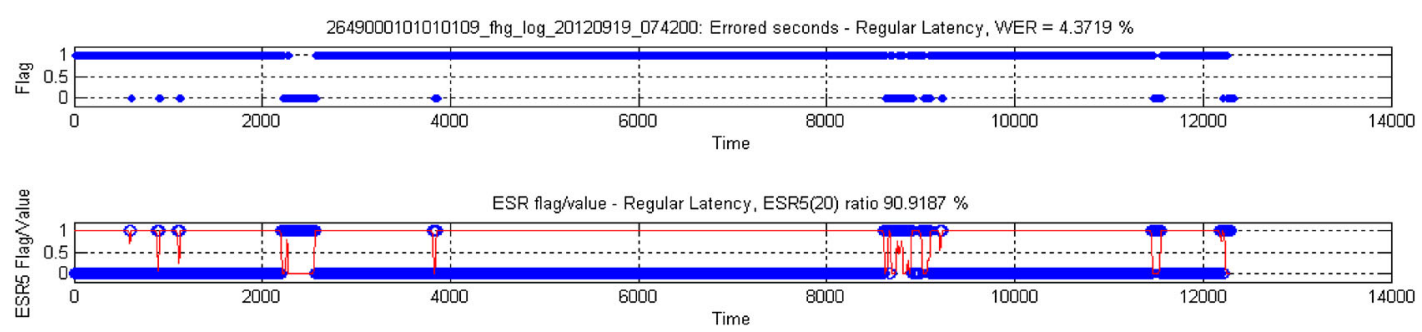

Figure 9. Time series of errored seconds (top) and time series of ESR5(20) flag from the test drive. 


\subsection{Return link analysis}

A preliminary validation of the return link has been carried out in [10] for open environment to quantify the static and mobile performance of the E-SSA transmission system in open environment. While the validation in [10] focuses on the physical layer, the following results provide link and IP layers statistics for the mobile environment. Within this paper, results are reported for two different mobile scenarios over the same itinerary covering multiple environments (as illustrated in Figure 6). The objective of performing twice the same itinerary is to compare the performance results in a fair manner (same environment). The first scenario is considered as the benchmark scenario as only one single user (OBU) transmits dummy IP packets with full power over the return link without background traffic. In such a scenario, the received packets at the hub are directly decoded by the demodulator, and no successive interference cancellation (SIC) cycle is executed. Instead, a more realistic case is envisaged in the second scenario as, in addition to the dummy IP packets transmitted by the OBU, the background traffic is activated. As shown in Figure 1, the traffic emulator will transmit E-SSA bursts directly toward the satellite and emulate a traffic load of 2000 packets per second $(0.96 \mathrm{~b} / \mathrm{s} / \mathrm{Hz})$. This value represents the highest traffic load supported by the implemented prototype platform at the time of the field trials. Further works are being carried out in order to reach higher traffic load as specified in [4]. Finally the Tx open loop power control algorithm [1] of the OBU has been enabled in the second scenario.

The link and physical layer configurations used during these two trials are detailed in Tables I and II, respectively. In both scenarios the OBU transmitted messages of 1200 bits through a dummy application layer which generated same size IP packets and delivered them to the OBU link layer. On the hub side, three nodes have been set up in cascade so as to allow up to 12 SIC cycles for scenario 2 .

In order to obtain reliable statistics, a considerable number of packets had to be transmitted. This goal has been achieved by selecting a link layer configuration that allowed a high transmission rate as seen in Table I. As the use of packets that require acknowledgment reduces the transmission rate (due to the acknowledgment time-out that can take up to $25 \mathrm{~s}$ as experienced during the field trials),

Table I. Link layer configuration.

\begin{tabular}{ll}
\hline Configuration & \multicolumn{1}{c}{ Scenario 1 and Scenario 2} \\
\hline IP fragmentation & No (1 IP packet =1 fragment) \\
Class of Service (CoS) & $1(50 \%)$ and 2 $(50 \%)$ (randomly distributed) \\
acknowledgment (ACK) type & noACK $(97 \%)$ and crcACK (3\%) (independent from CoS) \\
Persistence & 1 \\
ACK time-out & $25 \mathrm{~s}$ \\
Back Off Time (BOT) & $500 \mathrm{~ms}$ \\
Retransmission & No \\
\hline
\end{tabular}

Table II. Physical layer configuration.

\begin{tabular}{lccc}
\hline Configuration & Scenario 1 & Scenario 2 & Unit \\
\hline Itinerary & 3 & 3 & hours \\
Max OBU power (electrical) & 3 & 3 & $\mathrm{dBW}$ \\
Tx-control active & No & Yes & $\mathrm{dBi} / \mathrm{K}$ \\
G/T_OBU & NA & -25.00 & $\mathrm{dBW}$ \\
EIRP_DL & NA & 61.50 & $\mathrm{Mbaud}$ \\
FWD_Signal_BW & NA & 2.34 & $\mathrm{dBm}$ \\
N_SAT [1] & NA & -172.00 & $\mathrm{~dB}$ \\
K [1] & NA & 8.00 & $\mathrm{~dB}$ \\
R_max [1] & NA & 0.00 & $\mathrm{~dB}$ \\
Back traffic & No & Yes & $\mathrm{dBW}$ \\
Back traffic power spread & NA & 10.5 & pkts/s \\
Back traffic max power (uniform distribution) & NA & 2000 & \\
Back traffic load & NA & & \\
\hline
\end{tabular}

OBU, On-Board Unit; Max, maximum; NA, not applicable. 
it has been decided to transmit mostly packets that did not request acknowledgments (only $500 \mathrm{~ms}$ of waiting time between two consecutive packets). This long measured acknowledgement reception is due to some limitations in the prototype at the hub. For future commercial systems, a much lower acknowledgment time-out shall be considered.

Performance results of both scenarios are summarized in Table III. The two main metrics considered are on the one side the Packet Loss Ratio (PLR) and on the other side the Fragment Loss Ratio (FLR). These two ratios quantify the packet and fragment losses on the return link transmission at IP and link layers, respectively.

Focusing first on scenario 1, although the OBU transmitted at full power, the corresponding FLR reached $5.29 \%$. This value can be explained on the one hand by the few deep fade events encountered during the journey and on the other hand, by the use of two different antennas for Rx and Tx. In our setup, the satellite availability is estimated by monitoring the received signal level of the forward link at the Rx antenna, and the OBU is allowed to transmit only if the satellite is available. By consequent, as the Tx antenna is almost $2 \mathrm{~m}$ distant from the Rx antenna on the roof of the vehicle, it may happen that the satellite is seen as available for the reception, but not for the transmission (different channel environment). Moreover, it must be noted that both Rx and Tx antennas are already in another channel condition during the transmission time interval $(250 \mathrm{~ms})$ due to mobility of the vehicle. Nevertheless, these effects shall be mitigated by the use in the future of one single antenna for Tx and Rx, achieving thus a lower FLR. Potential optimization could be also reached by regulation of the instantaneous transmission power along the E-SSA burst time interval instead of setting a constant power level for the complete transmission burst in advance.

Keeping the focus on scenario 1, the main reason why the PLR deviates from the FLR lies in the satellite unavailability caused by the few intermediate and heavy tree shadowing events encountered during the field trials. In such events, as the application layer does not obtain satellite information, it still forwards the packets to the link layer, although the latter does not forward them to the physical layer (no buffer has been used at link layer level for this trial). The FLR is thus not affected by the satellite unavailability whereas the PLR is. As the two scenarios yield similar results, the same conclusion can be drawn for scenario 2 .

Comparing now the performance results of scenario 2 with respect to scenario 1 , it can be observed that both ratios (FLR and PLR) are slightly greater in the second scenario. The cause of this minor deviation is manifold. As described previously, it lies first of all in the distance between both antennas on the roof of the vehicle. It may happen that the Rx antenna faces good channel conditions but the Tx antenna does not. In such case, as the Rx signal level of the forward link is good and the power control algorithm of the $\mathrm{OBU}$ is activated, the $\mathrm{OBU}$ will transmit with a lower power, which results in a loss of the fragments due to channel fading. Conversely, if the Rx antenna is in a deep fade, the computed Tx power may exceed the terminal capability of $3 \mathrm{dBW}$, although the channel conditions are sufficient for transmission. Second, this deviation might be due to the limited number of serial interference cancellation

Table III. Return link performance results.

\begin{tabular}{lcr}
\hline Results & Scenario 1 & Scenario 2 \\
\hline OBU & & 5736 \\
\# IP transmitted & 5303 & 5370 \\
\# frag sent to the PHY: & 5027 & 366 \\
\# frag not sent to the PHY: & 276 & 5011 \\
HUB & 4761 & 12.64 \\
\# frag received & & 6.68 \\
Overall & 10.22 & -7.4 \\
IP PLR (\%) & 5.29 & -4.31 \\
Link layer FLR (\%) & 3 & 2.95 \\
Tx power min (dBW) & 3 & 1.71 \\
Tx power mean (dBW) & 3 & 0 \\
Tx power max (dBW) & 0 & \\
Tx standard deviation (dB) & & \\
\hline
\end{tabular}

OBU, On-Board Unit; IP, Internet Protocol; PLR, Packet Loss Ratio; FLR, Fragment Loss Ratio. 
iterations in the E-SSA demodulator, limited to 12 SIC cycles for this trial. Third, the share of fragments not sent to the physical layer (PHY) is slightly higher in scenario $2(6 \%)$ with respect to scenario $1(5 \%)$ due to minor 'physiological' changes in the route, such as a longer or shorter stop at a traffic light.

As to the packet transmission latency, Figure 10 shows the service delivery delay (SDD, [1]) for both scenarios. It can be observed that the SDD performance worsens with the background interference (scenario 2) as SIC cycles need to be executed to remove interference power and decode the received packets. However, the SDD remains below the theoretical latency boundary of $15 \mathrm{~s}$ for scenario 2 (the demodulation latency will not exceed $5 \mathrm{~s}$ per node even with the presence of high traffic).

Concerning the Tx power, Figure 11 illustrates the Tx power distribution of the OBU in scenario 2. The resulting spread is $10.35 \mathrm{~dB}$, which is in line with the one used for the background traffic. The difference in the absolute value is meant to compensate the Gain to system noise temperature ratio $(\mathrm{G} / \mathrm{T})$ difference between the locations of the field trials (center of coverage, Germany) and of the traffic emulator (edge of coverage, France). With the Tx power control activated, the terminal Effective Isotropically Radiated Power turns out to be, on average, $7 \mathrm{~dB}$ less than the case without the power control, which, notwithstanding a background traffic load of $2000 \mathrm{pkts} / \mathrm{s}$, allowed the achievement of almost the same PLR and FLR performances with respect to scenario 1. This shows the remarkable behavior of the Tx control algorithm, which has the main goal of avoiding transmissions in presence of extremely deep fading in order not to result in a waste of power and a source of harmful interference to the other terminals.

\section{POTENTIAL USE OF S-BAND MOBILE INTERACTIVE MULTIMEDIA: THE SAFETRIP PLATFORM}

A potential use of the S-MIM standard for end-to-end system has been implemented in the SafeTRIP project, whose general objective is to improve the use of road transport infrastructures and the alert chain (information/prevention/intervention) in case of incidents by offering an integrated system platform for data collection to service provisioning [9]. SafeTRIP benefits from the S-band satellite technology, which is optimized for two-way communication for OBU vehicles. Within this project, the satellite return channel makes use of the S-MIM standard to provide messaging services over GEO satellite by using low-power terminals (OBU). S-MIM has been integrated into SafeTRIP trying to make it as much transparent as possible to end-user applications. In order to achieve this goal, a dedicated middleware has been developed to act as separation layer between the end-user applications and the S-MIM and DVB-SH communication layers.

In-vehicle applications within SafeTRIP can send messages to service centers connected to the Internet using a very simple Simple Object Access Protocol API provided by the so-called Service Enabling Layer (SEL). Messages are then formatted by the SEL, which transmits them to the link layer. S-MIM is then used to deliver content via satellite to the HUB. At the HUB side the link layer components take care of decapsulating S-MIM messages and delivering to the ground middleware system (Service Enabling Platform or SEP). The SEP finally dispatches messages to service centers.
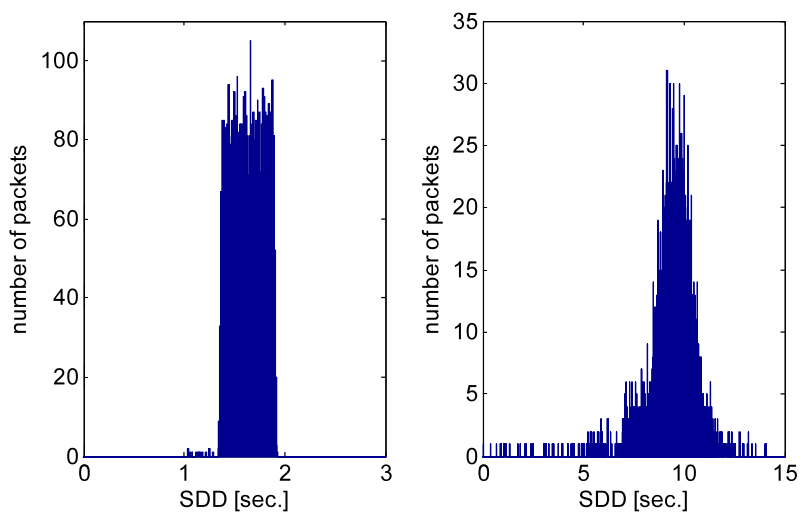

Figure 10. Service delivery delay (SDD) histogram for scenario 1 (left) and scenario 2 (right). 


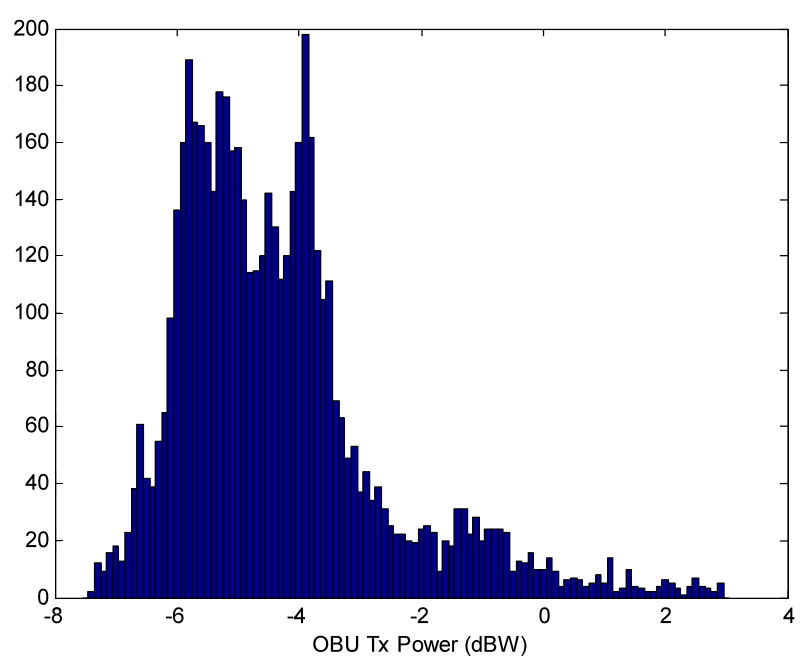

Figure 11. On-Board Unit transmission (electrical) power distribution.

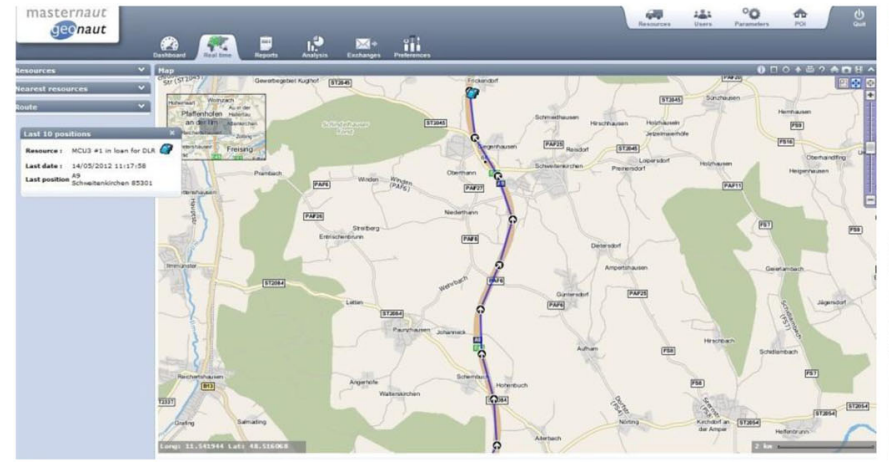

(a)

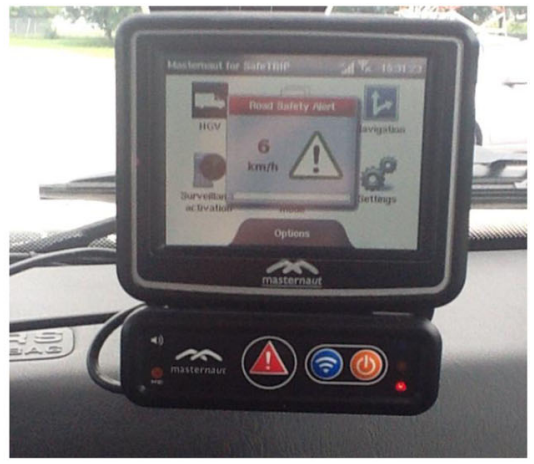

(b)

Figure 12. Real time tracking (a) and received collaborative road alert (b) - Courtesy of Masternaut.

On the forward link, service centers send messages using the SEP that dispatches them over the DVB-SH channel. On the vehicle, the SEL receives and filters messages over the forward link and delivers them to the end-user application.

Among the SafeTRIP applications making use of the S-MIM standard, the real time tracking of the vehicle as well as the collaborative road alert are briefly presented in this paper. The former transmits regularly its position to the control center over the return satellite channel, allowing thus a constant tracking of the vehicles. The latter permits the SafeTRIP vehicle to declare a danger to the whole community by sending an alert via the S-MIM return channel that will be then broadcasted to all other vehicles. Figure 12 illustrates the real time tracking application as seen in the control center (a) as well as the received collaborative road alert on the Human Machine Interface installed in the SafeTRIP vehicle (b).

\section{CONCLUSIONS}

A first implementation of the S-MIM technology was realized within the SafeTRIP project, allowing the validation of both forward and return link. The field trials carried out within this work were performed under real mobile channel environments (highway, tree shadowing, suburban, etc.), in such a way that the comparison of the two considered scenarios could be as fair as possible. 
This work allowed presenting results in terms of performances, which can be deemed satisfactory: the DVB-SH reception has been proved reliable and the different loss ratios (PLR, FLR, etc.) over the return link are in line with the expected results in a mobile environment characterized by deep fades. Further, this work confirmed the effectiveness of the S-MIM messaging protocol and more particularly, of the Tx power control algorithm, whose introduction permits a smart use of the satellite bandwidth, allowing the transmission of thousands of packets at moderate emitting power, hence proving its appropriate behavior, which is crucial for the development of low-cost consumer products.

\section{ACKNOWLEDGMENTS}

The authors of this paper would like to thank the partners within the SafeTRIP consortium. They are in particular thankful to the FP7 and the European Commission for funding the SafeTRIP project.

\section{REFERENCES}

1. ETSI TS 102 721. Satellite Earth Stations and Systems (SES); air interface for S-band Mobile Interactive Multimedia (S-MIM), V1.1.1.

2. ETSI TS 102 585. Digital Video Broadcast (DVB); System Specifications for Satellite services to Handheld devices (SH) below $3 \mathrm{GHz}, \mathrm{V} 1.2 .1$

3. De Gaudenzi R, Elia C, Viola R. Bandlimited quasi-synchronous CDMA: a novel satellite access technique for mobile and personal communications systems. IEEE Journal in Selected Areas in Communications 1992; 10(2):328-343.

4. de Gaudenzi R, Del Rio Herrero O. High efficiency satellite multiple access scheme for machine-to-machine communications. IEEE Transactions on Aerospace and Electronic Systems 2012; 48(4):2961-2989.

5. Del Río Herrero O, De Gaudenzi R. Methods, apparatuses and system for asynchronous spread-spectrum communication. European Patent Application nr. 08290801.3, August 2008.

6. Del Río Herrero O, De Gaudenzi R. A high efficiency scheme for quasi-real-time satellite mobile messaging systems. 10th Signal Processing for Space Communications, October 2008.

7. Scalise S, Párraga Niebla C, Arcidiacono A, Finocchiaro D, De Gaudenzi R, Del Rio Herrero O, Schlüter G, Krause J. S-MIM: a novel radio interface for efficient messaging services over satellite. Workshop on Telecommunications: From Research to Standards, IEEE ICC, 2012.

8. Scalise S, Niebla CP, Gallinaro G, Andrenacci M, Rinaldo R, Del Rio Herrero O, Breiling, M, Finocchiaro D, Cebrian Puyuelo JM, Schlüter G. System design for pan-European MSS services in S-band. Advanced Satellite Multimedia Systems Conference (ASMA) and the 11th Signal Processing for Space Communications workshop (SPSC), 13-15 Sept. 2010, 5th vol.; 538, 545.

9. SAFETRIP Project webpage. http://www.safetrip.eu [accessed on: October 2009].

10. Heyn T, Nagel P, Niemann B, Recchia A, Collard F, Hermenier R, Marchitti MA, Andrenacci M, Mendola G. E-SSA satellite messaging return channel: performance analysis and field trials results. 6th Advanced Satellite Multimedia Systems Conference, 5-7. Sept.2012, Baiona, Spain.

11. ETSI TS 103 179. Satellite Earth Station and Systems: Return Link Encapsulation protocol (RLE), V0.0.3

12. http://www.eutelsat.com/en/satellites/the-fleet/EUTELSAT-10A.html [accessed on: April 2009].

13. Recchia A, Collard F, Antip N. Performance of the S-MIM messaging protocol over satellite. 6th Advanced Satellite Multimedia Systems Conference, 5-7. Sept.2012, Baiona, Spain.

14. Andrenacci M, Mendola G, Collard F, Finocchiaro D, Recchia A. E-SSA demodulator implementation, laboratory tests and satellite validation. Special Issue Paper, submitted to International Journal of Satellite Communications and Networking, 2013

15. ETSI TS 102 584. Digital Video Broadcast (DVB); DVB-SH Implementation Guidelines, V1.3.1

\section{AUTHORS’ BIOGRAPHIES}

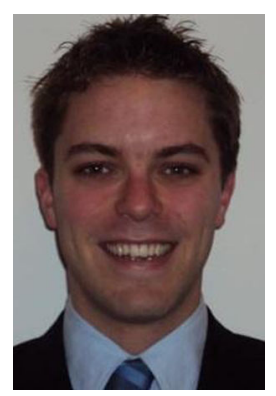

Romain Hermenier studied at the Institut National des Télécommunications (Telecom SudParis) in Evry, France, from 2005 to 2007 and at the Institut Supérieur de l'aéronautique et de l'espace (Supaero), in Toulouse, France, in 2008, where he specialized in the field of satellite communications. He received his Master of Engineering from Telecom SudParis in 2008. Since 2008, he is working as a scientific researcher and project manager (PMP certified) in the Institute of Communications and Navigation at the German Aerospace Center (DLR). He is currently involved in S-Band project activities related to the S-band Mobile Interactive Multimedia (S-MIM) standard. 


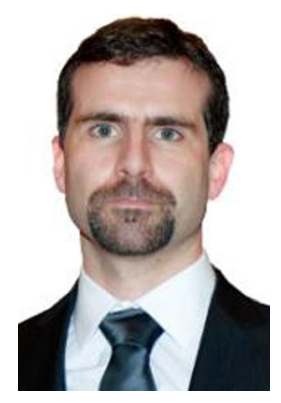

Alessandro Del Bianco studied at the University of Bologna, where he graduated the Bachelor's degree in 'Telecommunications Engineering' in 2005 and Master's degree in 'Electronic and Telecommunications Engineering' in 2007. Since 2007, he is working as a scientific researcher at DLR, and his main involvements are in the field of satellitemobile communication systems.

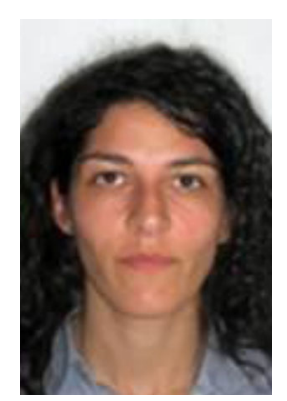

Maria Antonietta Marchitti received the Laurea degree in 'Telecommunications Engineering' from the University of Cassino (Italy), in 2004. In 2005, she received the MSc in 'Advanced Communications and Navigations Satellite Systems' from Tor Vergata University (Rome, Italy). From September 2005 to June 2008, she was with Aalborg University, Denmark, where she was involved in the design of mobility and security solutions for B3G mobile systems. After a short experience as software engineer in the development of IP solutions for mobile phones with Tieto (Arhus, Denmark), in May 2010, she has joined the Institute of Communications and Navigation at the German Aerospace Center (DLR), where her main research interests are cooperative routing protocols with emphasis on satellite networks.

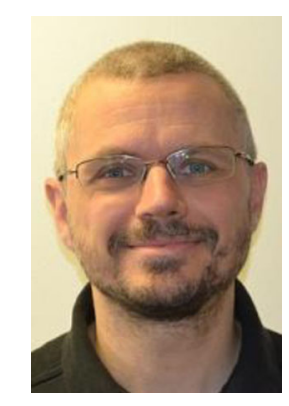

Thomas Heyn was born in 1970. He received his degree in Electrical Engineering (Dipl. Ing.) from the Friedrich-Alexander-University in Erlangen in 1996. He joined Fraunhofer Institute for Integrated Circuits in 1996 where he has been working in national and international projects in the area of telecommunication and digital broadcasting systems for contracting authorities and industrial partners. He is the head of the Applied Communications Group and member of the Integral SatCom Initiative - European Technology Platform. His current research interests include future mobile networks investigating LTE technologies $4 \mathrm{G}$ and beyond.

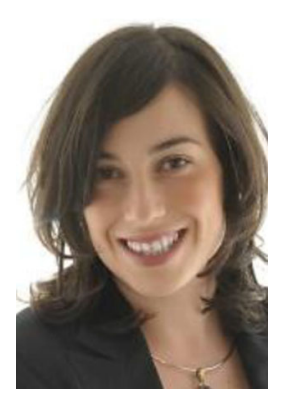

Annamaria Recchia received a Master Degree in Telecommunications Engineering from 'Politecnico di Torino', with a thesis on 'Performance assessment of an efficient satellite mobile messaging system', based on the Enhanced Spread Spectrum Aloha (E-SSA) protocol, standardized as S-MIM (Part 3) at ETSI. Her recent activities have been mainly related to the implementation and optimization of the E-SSA satellite system. She has taken part as technical expert to several collaborative projects, for example, the SISTER project and the SafeTRIP project, both EC funded. Since April 2013, she has joined the Pre-Sales Team within Eutelsat Commercial Department. 


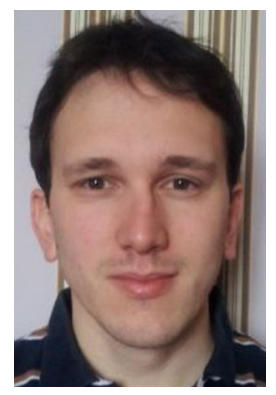

Florian Collard received an Engineering degree from TELECOM Bretagne. He finalized his degree with the specialized master 'Space Communication Systems' in 'Institut Supérieur de l'Aéronautique et de l'Espace' in Toulouse. Since 2010, he did a PhD in collaboration with Eutelsat (Innovation team) and ISAE (SCANR team). His research focuses on the analysis and the optimization of the Enhanced Spread Spectrum Aloha. He was involved in the first trials of the S-MIM protocol over satellite. He is now involved in 'smart LNB' development in Eutelsat.

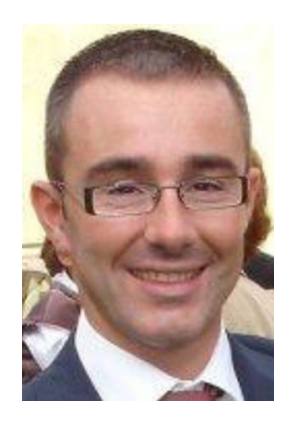

Marco Andrenacci received his Bachelor's Degree in Telecommunication Engineering at the Pisa University in 1997. Since April 2008, he has been Project Manager at MBI S.r.L. (Pisa, Italy) where he coordinates the S-band development activities. He has been the technical leader for MBI in several ESA and EU S-band projects (SAFETRIP, SATURN, DENISE, MireSys, and J-Ortigia). He has over 10 years of experience in designing and deploying telecommunication networks with heterogeneous transport technologies.

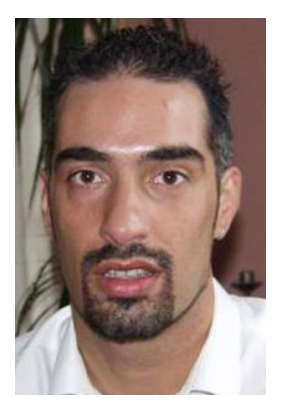

Gaetano Mendola is a Principal Software Engineer in MBI, he is in charge of SDR related developments, and his areas of interest are high performance computing, GPGPU computing, parallel computing, and big data.

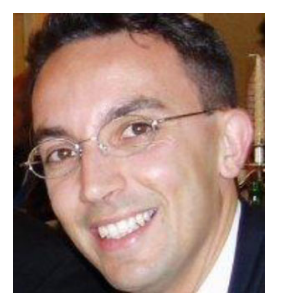

Attilio Vaccaro is currently a Senior System Architect at MBI Srl. He is experienced in satellite communication systems design and implementation mainly for ICT and ITS fields. $\mathrm{He}$ is currently working on implementation of space technologies for agriculture and M2M systems. 